\title{
Diagnósticos de enfermagem relacionados à amamentação em unidade de alojamento conjunto
}

\author{
Nursing diagnoses related to breastfeeding in a rooming-in unit
}

Diagnósticos de enfermería relacionados a la lactancia materna en unidad de alojamiento conjunto

\section{Evilene Pinto da Silva', Albertisa Rodrigues Alves", Ana Ruth Monteiro Macedo"', Regina Maria de Sá Barreto Bezerra", Paulo César de Almeida"', Edna Maria Camelo Chaves" \\ I Instituto Dr. José Frota. Fortaleza-CE, Brasil. \\ " Governo do Estado do Ceará, Secretaria da Saúde do Estado do Ceará, Hospital Geral de Fortaleza. Fortaleza-CE, Brasil. \\ I"' Universidade Estadual do Ceará, Centro de Ciências da Saúde, Departamento de Saúde Pública. Fortaleza-CE, Brasil.}

\section{Submissão: 12-06-2011 Aprovação: 15-03-2013}

\section{RESUMO}

O estudo objetivou identificar, em uma unidade de Alojamento Conjunto, diagnósticos de enfermagem relacionados ao fenômeno da Amamentação de acordo com a taxonomia II da NANDA-I. Realizou-se um estudo descritivo-exploratório, envolvendo 83 mães e seus bebês, no período de fevereiro a abril de 2011. Os dados foram coletados por meio de um instrumento fundamentado na Teoria das Necessidades Humanas Básicas e, após tratamento estatístico, apresentados em tabelas. Nos resultados, o diagnóstico mais frequente foi Amamentação eficaz, com 65 (78,3\%). Estima-se que os diagnósticos de enfermagem relacionados à Amamentação contribuirão para que os cuidados de enfermagem possam ser mais direcionados, de modo a promover uma assistência mais qualificada, humanizada e eficaz.

Descritores: Recém-Nascido; Mães; Diagnóstico de Enfermagem; Enfermagem; Aleitamento Materno.

\begin{abstract}
The study aimed to identify, in a Rooming-in unit, diagnoses related to the phenomenon of breastfeeding according to the NANDA-I Taxonomy II. It was conducted a descriptive exploratory study involving 83 mothers and their babies in the period of February to April 2011. Data were collected by means of an instrument based on the Theory of Basic Human Needs and, after statistical analysis, presented in tables. The results showed that the most frequent nursing diagnosis was Effective breastfeeding, identified in $65(78.3 \%)$ cases. It is estimated that the nursing diagnoses related to breastfeeding can contribute to nursing care so that it can be more targeted, in order to promote a more qualified, effective and human care.
\end{abstract}

Key words: Infant Newborn; Mothers; Nursing Diagnosis; Nursing; Breast Feeding.

\section{RESUMEN}

El objetivo del estudio fue identificar, en una unidad de Alojamiento Conjunto, los diagnósticos de enfermería relacionados con la Lactancia materna, así como sus factores relacionados y características definitorias. Realizó-se un estudio descriptivo, exploratorio, involucrando 83 madres y sus bebés, de febrero a abril de 2011. Los datos fueran recogidos por medio de un instrumento basado en la Teoría de las Necesidades Humanas Básicas, presentados en tablas estadísticas. En los resultados, el diagnóstico más frecuente fue la Lactancia materna eficaz, con 65 (78,3\%) casos. Se estima que los diagnósticos de enfermería relacionados con la Lactancia materna contribuyen para que la atención de enfermería pueda ser más específica con el fin de promover una asistencia más calificada, humana y efectiva.

Palabras clave: Recién Nacido; Madres; Diagnóstico de Enfermería; Enfermería; Lactancia Materna. 


\section{INTRODUÇÃO}

O leite materno é o alimento essencial e mais completo para a criança, pois é o único que oferece substâncias e nutrientes que esta precisa para crescer e se desenvolver com saúde. O tempo preconizado para o Aleitamento Materno Exclusivo (AME) é de seis meses, podendo se prolongar até dois anos junto com outros alimentos ${ }^{(1)}$.

Essa prática possui importância fundamental para a saúde da criança, já que apresenta valores nutricionais e imunológicos ao crescimento e desenvolvimento infantil, oferecendo também benefícios psicológicos para o binômio mãe-filho, auxiliando na formação do vínculo afetivo entre ambos ${ }^{(2)}$.

Ademais, o aleitamento materno também apresenta vantagens para a mulher, pois auxilia no fortalecimento do vínculo afetivo com o bebê, ajuda na involução uterina, atua na diminuição do risco de hemorragia, contribui ainda para o retorno ao peso anterior ao da gestação, além de auxiliar no intervalo entre as gestações ${ }^{(1)}$.

Para a criança, são muitas as vantagens da amamentação, pois recebe alimento completo, sem necessidade de qualquer acréscimo até os seis meses de idade, além de ajudar na excreção do mecônio e de conferir proteção contra infecções ${ }^{(1)}$. É válido ressaltar que o aleitamento materno tem fundamental importância na promoção do contato entre mãe e filho, fortalecendo, assim, o vínculo entre ambos ${ }^{(3)}$.

Diante disso, os enfermeiros devem incentivar o aleitamento materno e apoiar as mães para iniciá-lo o mais precocemente, auxiliando-as a adquirir autoconfiança em seu potencial para amamentar o filho ${ }^{(4)}$.

Tendo em vista que a Enfermagem presta assistência juntamente a uma equipe multidisciplinar que se encontra capacitada para desenvolver a atenção humanizada ao binômio mãe-filho, torna-se significante o incentivo ao aleitamento materno, com vistas ao melhor desenvolvimento da criança e a promoção do apego eficaz.

Devido ao número insuficiente de enfermeiros, ou ao excesso de atividades administrativas, ocorrem lacunas na assistência ao binômio mãe-filho, que podem resultar em uma atuação pouco expressiva ou ausente do enfermeiro na assistência ao aleitamento materno durante o pós-parto ${ }^{(5)}$. Contudo, as ações de enfermagem relacionadas ao aleitamento materno devem ser direcionadas e efetivas. Estas podem ser realizadas por intermédio da Sistematização da Assistência de Enfermagem (SAE) ou do Processo de Enfermagem (PE).

A Sistematização da Assistência de Enfermagem é relevante para a coordenação das atividades do enfermeiro, sendo útil tanto nas tarefas administrativas quanto nas assistenciais, dinamizando as ações de enfermagem. O Processo de Enfermagem (PE) auxilia o enfermeiro na organização e sistematização da prática de enfermagem, intensificando a valorização e o reconhecimento da enfermagem enquanto ciência ${ }^{(5-6)}$.

Destaca-se que a insuficiente utilização de uma linguagem universal para descrever a prática profissional tem comprometido o desenvolvimento da Enfermagem como ciência ${ }^{(7)}$. Neste sentido, observa-se a relevância da padronização da assistência de enfermagem por meio do Processo de Enfermagem.
A responsabilidade de cuidar em enfermagem exige que as decisões sobre as intervenções propostas sejam fundamentadas na avaliação do estado de saúde do indivíduo. Para tanto, esta avaliação requer a adoção do diagnóstico de enfermagem como referência para que ações de enfermagem sejam executadas $^{(8)}$. A assistência sistematizada representa uma opção adequada, pois oportuniza um cuidado mais individualizado, segundo as necessidades do cliente $^{(9)}$.

A NANDA Internacional (NANDA-I) define diagnóstico de enfermagem como o "julgamento clínico das respostas do indivíduo, da família ou comunidade a problemas de saúde/ processos vitais reais ou potenciais, proporcionando base para a seleção de intervenções de enfermagem para atingir resultados pela quais o enfermeiro é responsável"(10).

O diagnóstico de enfermagem é útil para estruturar o conhecimento da Enfermagem, direcionar a necessidade de cuidados do paciente e definir o papel do enfermeiro. A atividade diagnóstica aproxima o enfermeiro de seus clientes, facilitando, assim, o desenvolvimento de sua assistência, ao mesmo tempo em que se constitui em um instrumento facilitador das ações de enfermagem, uma vez que apontam as devidas intervenções de acordo com a necessidade do paciente ${ }^{(8-11)}$.

Assim, ressalta-se que a promoção da qualidade da assistência de enfermagem, através dos DE, compromete toda a equipe de enfermagem, que deverá empenhar-se com o objetivo de atender às necessidades dos pacientes, possibilitando a implementação de intervenções sistematizadas, contribuindo para melhoria da assistência ${ }^{(12-13)}$.

$\mathrm{O}$ interesse em desenvolver um estudo que permitisse a identificação de diagnósticos de enfermagem relacionados ao aleitamento materno no alojamento conjunto deu-se em razão importância da sistematização do cuidar durante o processo de amamentação, uma vez que a amamentação constitui-se num processo complexo de interação mãe e filho.

Os diagnósticos de enfermagem relacionados à amamentação, de acordo com a taxonomia II da NANDA-I (2009-2011) são: amamentação eficaz, amamentação ineficaz, amamentação interrompida. Na estrutura da taxonomia II da NANDA-I, os diagnósticos supracitados são categorizados como Diagnósticos Reais pertencentes ao Domínio 7: Papéis e relacionamentos; Classe 3: Desempenho de papéis ${ }^{(10)}$.

Amamentação eficaz é definida como a adequada proficiência e satisfação com o processo de amamentação para o binômio mãe-filho. Amamentação ineficaz representa a insatisfação ou dificuldade que a mãe, bebê ou criança experimenta com o processo de amamentação. Amamentação interrompida consiste na quebra do processo de amamentação como resultado de incapacidade ou inconveniência de colocar a criança no peito para mamar ${ }^{(10)}$.

Para o processo de julgamento clínico dos diagnósticos reais, faz-se necessário a interpretação da definição do enunciado diagnóstico, características definidoras e fatores relacionados, uma vez que o enfermeiro deve se preocupar com a existência de riscos à exatidão das interpretações ${ }^{(10)}$.

O enunciado e definição diagnóstica oferecem uma descrição clara e exata do enunciado que ajuda ao enfermeiro distinguir de outros diagnósticos similares. As características 
definidoras são indícios/inferências passíveis de observação, agrupadas como manifestações clínicas de todos os Diagnósticos Reais, ou seja, da situação diagnóstica (Ex: sucção no peito regular). Os fatores relacionados mostram uma relação padronizada com o diagnóstico de enfermagem, e estes geram a elaboração das intervenções de enfermagem individualizadas (Ex: estrutura oral da criança normal) ${ }^{(10)}$.

Diante do exposto, percebeu-se a necessidade da identificação dos DE relacionados ao aleitamento materno na unidade de Alojamento Conjunto (AC), uma vez que este estudo pode contribuir para a elaboração de uma assistência individualizada de acordo com as necessidades da mãe e filho. Objetivou-se identificar os diagnósticos de enfermagem relacionados à amamentação em recém-nascidos de uma unidade de Alojamento Conjunto, de acordo com a taxonomia II da NANDA-I ${ }^{(10)}$.

\section{METODOLOGIA}

Trata-se de pesquisa exploratório-descritiva, com abordagem quantitativa, desenvolvida em uma Unidade de Alojamento Conjunto (AC), de um hospital público estadual, em Fortaleza-CE, credenciado pelo Sistema Único de Saúde, que tem o título de Hospital Amigo da Criança.

A Unidade de Alojamento Conjunto é uma unidade com enfermarias com dois ou quatro leitos, com pias, bancada para higienização dos recém-nascidos. Atualmente, encontram-se credenciados 32 leitos. Prestam assistência por turno um Enfermeiro e um Técnico de Enfermagem para cada 15 recém-nascidos. Sessões de orientação sobre aleitamento materno são oferecidas diariamente pelos membros da equipe de enfermagem.

A população foi composta por 240 mães com bebês admitidas no AC. A amostragem, por conveniência, incluiu 83 mães, admitidas no AC, de acordo com a demanda do serviço, no período de fevereiro a abril de 2011, e que se encontravam com os filhos no processo de amamentação. Foram excluídas mães portadoras de HIV, recém-nascidos com idade gestacional menor do que 35 semanas e mães portadoras de distúrbios psiquiátricos.

As mães foram contatadas no AC pela pesquisadora e convidadas a participarem do estudo. Utilizou-se para a coleta de dados formulário semiestruturado, fundamentado na Teoria das Necessidades Humanas Básicas de Horta ${ }^{(14)}$, que serviu de roteiro para realização da anamnese e do exame físico da puérpera e do recém-nascido (RN). A partir dos dados coletados, realizou-se o julgamento clínico para a identificação dos diagnósticos de enfermagem relacionados com a Amamentação, bem como os fatores relacionados e características definidoras, baseando-se na taxonomia II da NANDA-I ${ }^{(10)}$.

Atualmente, o sistema de classificação de Diagnósticos de Enfermagem, da Taxonomia II, da NANDA-I ${ }^{(10)}$, tem sido o mais utilizado na prática clínica hospitalar, uma vez que proporciona a base para seleção de intervenções de enfermagem para alcançar resultados pelos quais a enfermeira é responsável.

A pesquisa foi desenvolvida em consonância com a Resolução 196/96, do Conselho Nacional de Saúde/Ministério da Saúde que discorre sobre a pesquisa envolvendo seres humanos, após aprovação do Comitê de Ética em Pesquisa, conforme número 091202/10. Cada participante assinou um Termo de Consentimento Livre Esclarecido (TCLE), sendo informado acerca da garantia do sigilo que assegurava a privacidade quanto aos dados confidenciais envolvidos na pesquisa.

Após identificação dos DE, os dados foram organizados e apresentados em tabelas com frequência absoluta e percentual, e discutidos conforme literatura pertinente ao tema.

\section{RESULTADOS}

Tabela 1 - Descrição das características das puérperas de uma unidade de Alojamento Conjunto. Fortaleza, CE, 2011.

\begin{tabular}{lccl}
\hline Características & N & $\%$ & $\begin{array}{l}\text { Média/ } \\
\text { Desvio Padrão }\end{array}$ \\
\hline Idade (anos) & & & \\
$\quad 14-19$ & 25 & 30,2 & Média $=24$ anos \\
$\quad 20-34$ & 47 & 56,6 & DP \pm 7anos \\
$\quad$ Acima de 35 anos & 11 & 13,2 & \\
& & & \\
Escolaridade & & & \\
$\quad$ Ensino Fundamental Incompleto & 41 & 49,4 & \\
$\quad$ Ensino Fundamental & 10 & 12,0 & \\
$\quad$ Ensino Médio Incompleto & 6 & 7,2 & Média $=2,24$ anos \\
$\quad$ Ensino Médio Concluído & 23 & 27,7 & DP $\pm 1,4$ anos \\
$\quad$ Ensino Superior & 3 & 3,6 &
\end{tabular}

Estado civil

Casada

União Consensual

Solteira

$21 \quad 25,3$

$44 \quad 52,4$

$18 \quad 21,4$
Realizou pré-natal na instituição de realização do estudo
Sim
Não

$51 \quad 60,7$

$32 \quad 39,3$

\section{Recebeu orientação sobre aleitamento materno \\ Sim \\ Não}

5363,9

3036,1

Total
83100,0
A amostra foi composta de 83 mães, com predominância na faixa etária de 20 a 34 anos, representando 47 (56,6\%), seguido da faixa etária de 14 a 19 anos, com 25 (30,2\%), a faixa etária acima de 35 anos esteve entre 11 (13,2 \%) mães participantes do estudo. A escolaridade foi diversificada, predominando o Ensino Fundamental incompleto, 41 (49,4\%), e Ensino Médio completo, com 23 (27,7\%) mães. Em relação ao estado civil, 44 (52,4\%) das mães encontravam-se em união consensual, seguido do estado civil casada, com 21 $(25,3 \%)$. A maioria das mães realizou consulta de pré-natal na instituição de realização do estudo, 51 (60,7\%). O total 
de mães que recebeu orientação sobre aleitamento materno foi de $53(63,9 \%)$, portanto, constituindo a maioria das mães.

Tabela 2 - Descrição das características dos recém-nascidos em uma unidade de Alojamento Conjunto. Fortaleza, CE, 2011.

\begin{tabular}{|c|c|c|c|}
\hline Características & $\mathbf{N}$ & $\%$ & $\begin{array}{l}\text { Média } \\
\text { Desvio Padrão }\end{array}$ \\
\hline \multicolumn{4}{|l|}{ Sexo } \\
\hline Masculino & 56 & 67,5 & \multirow{2}{*}{ - } \\
\hline Feminino & 27 & 32,5 & \\
\hline \multicolumn{4}{|l|}{ Parto } \\
\hline Cesárea & 54 & 65,1 & \\
\hline Normal & 29 & 34,9 & \\
\hline \multicolumn{4}{|c|}{ Idade gestacional (semanas) } \\
\hline $35-37$ & 21 & 25,3 & \\
\hline $38-42$ & 62 & 74,7 & \\
\hline \multicolumn{4}{|c|}{ Peso de nascimento (gramas) } \\
\hline Menor de 2.499 & 13 & 15,7 & $\mathrm{Me}=3.090$ \\
\hline Acima de 2.500 & 70 & 84,3 & $\mathrm{DP} \pm 590 \mathrm{~g}$ \\
\hline Total & 83 & 100,0 & \\
\hline
\end{tabular}

Em relação ao sexo dos recém-nascidos, predominou o masculino, com 56 (67,5\%). O parto cesáreo ocorreu em 54 $(65,1 \%)$ das mulheres, seguido do parto normal, 29 (34,9\%). A idade gestacional predominante foi de 38 a 42 semanas de gestação 62 (74,7\%). O nascimento de recém-nascido com peso acima de $2.500 \mathrm{~g}$ foram $70(84,3 \%)$.

Tabela 3 - Descrição dos Diagnósticos de Enfermagem em uma unidade de Alojamento Conjunto. Fortaleza, CE, 2011.

\begin{tabular}{lcc}
\hline Diagnósticos & N & \% \\
\hline Amamentação eficaz & 65 & 78,3 \\
Amamentação ineficaz & 11 & 13,3 \\
Amamentação interrompida & 7 & 8,4 \\
Total & 83 & 100,0 \\
\hline
\end{tabular}

O diagnóstico de enfermagem prevalente foi Amamentação eficaz, identificado em 65(78,3\%) dos casos, seguidos de Amamentação ineficaz 11(13,3\%) e Amamentação interrompida com $7(8,4 \%)$ dos casos.
Tabela 4 - Distribuição de frequência das características definidoras e dos fatores relacionados segundo diagnósticos de enfermagem. Fortaleza-CE, 2011.

\begin{tabular}{lcc}
\hline $\begin{array}{l}\text { Diagnóstico, características definidoras } \\
\text { e fatores relacionados }\end{array}$ & N & \% \\
\hline $\begin{array}{l}\text { Amamentação Eficaz } \\
\text { Características Definidoras }\end{array}$ & 65 & 100,0 \\
$\quad$ Criança está satisfeita após a mamada & 55 & 84,6 \\
$\quad$ Padrão de peso adequado & 43 & 66,1 \\
$\quad$ Deglutição no peito regular & 37 & 56,9 \\
$\quad$ Sucção no peito continua & 35 & 53,8 \\
$\quad$ Sucção no peito regular & 24 & 36,9 \\
$\quad$ Deglutição no peito continua & 23 & 35,3 \\
$\quad$ Mãe é capaz de posicionar a criança & 22 & 33,8 \\
$\quad$ Verbalização materna de satisfação & 16 & 24,6
\end{tabular}

Fatores Relacionados

$\begin{array}{lcc}\text { Estrutura oral da criança normal } & 65 & 100,0 \\ \text { Estrutura mamária normal } & 63 & 96,9 \\ \text { Idade gestacional acima de 34s } & 59 & 90,7 \\ \text { Confiança materna } & 14 & 21,5 \\ \text { mentação Ineficaz } & 11 & 13,3 \\ \begin{array}{l}\text { aracterísticas Definidoras } \\ \text { Criança exibe agitação }\end{array} & 11 & 100,0 \\ \begin{array}{l}\text { Criança chora ao ser posta no peito } \\ \text { Criança se arqueia no peito }\end{array} & 10 & 90,9 \\ \begin{array}{l}\text { Incapacidade da criança apreender } \\ \text { a região areolar-mamilar }\end{array} & 7 & 63,3 \\ \end{array}$

Fatores Relacionados

$\begin{array}{lll}\text { Ansiedade materna } & 8 & 72,7\end{array}$

Déficit de conhecimento $\quad 3 \quad 27,3$

$\begin{array}{lcc}\begin{array}{l}\text { Amamentação Interrompida } \\ \text { Características Definidoras }\end{array} & 7 & 100,0 \\ \text { Separação mãe-filho } & 5 & 71,4 \\ \text { Desejo da mãe de oferecer o seu leite } & 5 & 71,4\end{array}$

Fator Relacionado

Prematuridade

$6 \quad 85,7$

O DE Amamentação eficaz a característica definidora (CD) mais frequente foi a criança está satisfeita após a mamada $55(84,6 \%)$ e o fator relacionado (FR) foi a estrutura oral da criança normal 65 (100\%). O DE Amamentação ineficaz a CD 
mais frequente foi a criança exibe agitação 11 (100\%) e FR foi ansiedade materna, 8(72,7\%). A amamentação interrompida foi verificada em sete participantes, da qual teve como CD separação mãe-filho e desejo da mãe de oferecer o seu leite, $5(71,4 \%)$, e FR prematuridade, $6(85,7 \%)$.

\section{DISCUSSÃO}

Caracterizou-se a amostra como de puérperas jovens, ressaltando-se a presença de adolescentes $(30,2 \%)$ na amostra, o que representa um dado considerável, pois requer do serviço de pré-natal a disponibilidade de estratégias e tempo para o acompanhamento destas gestantes, a fim de melhorar os índices de $\mathrm{AME}^{(15)}$. Vale destacar que neste período, a mulher já vivenciou ajustamentos sociais e psicológicos, o que provoca mudanças, que influenciam e melhoram o autoconceito, pois se tratam de mães adultas, das quais possuem maturidade para cuidar de seus filhos.

Ademais, destaca-se que a maioria das puérperas não concluiu o Ensino Médio. Estudos apontam a escolaridade como fator para a dificuldade da amamentação ${ }^{(16-17)}$. As puérperas informaram que durante as consultas foram orientadas sobre a realização da amamentação. Vale ressaltar que, as mães que realizaram o pré-natal na instituição onde se realizou a pesquisa, participam de sessões educativas na sala de espera ministradas por enfermeiros do Núcleo de Aleitamento Materno (NUAM) e acadêmicos de enfermagem. Ao serem admitidas no AC, foram incentivadas e recebiam apoio da equipe de multiprofissional, em particular da enfermagem para amamentar.

Estudo realizado com bebês em idade gestacional de 35 a 37 semanas, considerados prematuros que se encontravam com suas mães no AC, identificou a idade gestacional como fator definidor para ocorrência da amamentação materna, pois bebês prematuros necessitam de maiores cuidados. Vale ressaltar a importância do apoio ao abordar a mãe sobre amamentação, pois o bebê pode apresentar dificuldades em relação à pega e coordenação de sucção, respiração e deglutição. Faz-se necessário fornecer informação positiva sobre o bebê, pois isto minimiza os sentimentos de ansiedade, frustração, incertezas e culpa relacionados à amamentação, tornando as puérperas menos vulneráveis ${ }^{(18)}$.

Convém referir que prematuros com funções vitais estabilizadas são encaminhados ao AC. Dos sete casos com DE Amamentação interrompida, seis apresentaram a prematuridade como fator relacionado.

$\mathrm{Na}$ unidade de Alojamento Conjunto, o aleitamento materno é incentivado e estimulado pelas inúmeras vantagens apresentadas tanto para mãe quanto para o filho. Este cuidado tem início nas primeiras horas de vida, o mais precocemente possível, com orientações adequadas sobre o $\mathrm{AM}^{(19)}$.

O peso foi um dos principais indicadores para avaliação do crescimento pós-natal. O baixo peso ao nascer (peso inferior à $2500 \mathrm{~g}$ ) foi o fator de risco mais comumente associado às mortes perinatais. Mais de $80 \%$ das crianças do estudo apresentaram peso acima de $2500 \mathrm{~g}$, portanto o processo de amamentação sofreu pouca ou nenhuma influência deste fator.

Quanto ao tipo de parto, identificou-se que a maioria das puérperas teve parto cesariano. Apesar da separação imediata entre mãe e filho que este tipo de parto oferece, além de outros fatores, como recuperação mais demorada e dolorosa, o parto cesariano não impossibilitou o aleitamento materno.

A Amamentação eficaz pode ser percebida quando o binômio demonstra adequada proficiência e satisfação com o processo de amamentação. Isto significa que mãe e o filho estão em harmonia, bem adaptados ao ambiente do Alojamento Conjunto, que tem como principal função promover esta interação. Este DE foi o mais presente na unidade de AC investigada. Assim, reforça-se que o leite materno nas primeiras semanas de vida é de grande importância, pois este representa fator de proteção a crianças de zero a cinco anos contra o sobrepeso(20).

O segundo DE mais frequente foi Amamentação ineficaz relacionado, principalmente, à ansiedade materna e ao déficit de conhecimento. Outro estudo comprovou que amamentação ineficaz esteve relacionada ao conhecimento deficiente ${ }^{(21)}$.

A Amamentação interrompida esteve presente quando houve descontinuidade no processo de amamentação. Esteve principalmente relacionada à prematuridade e caracterizada pela separação entre mãe e filho, além do desejo da mãe em oferecer o leite materno.

\section{CONCLUSÃO}

O desenvolvimento desta pesquisa favoreceu a identificação de DE relativos à amamentação em 83 mães e seus RN em uma unidade de alojamento conjunto de um hospital público estadual. O diagnóstico mais encontrado foi Amamentação Eficaz, seguido de Amamentação Ineficaz e Amamentação Interrompida.

O presente estudo ressaltou a importância do processo de elaboração e julgamento clínico dos diagnósticos de enfermagem relacionados à amamentação, uma vez que estes são a base para elaboração apropriada das intervenções e alcance dos resultados positivos na assistência de enfermagem à mãe e seu RN.

Considera-se a amamentação um processo que envolve desafios na interação mãe e filho, bem como exige dos profissionais de enfermagem qualificação para atuar no AC. De acordo com os resultados encontrados, este estudo evidenciou o sucesso da amamentação para a mãe e seu RN, uma vez que o DE que mais ocorreu foi Amamentação Eficaz, e isso pode ter sido resultado de ações educativas implementadas através de um programa específico para o incentivo ao aleitamento materno existente na referida instituição do estudo.

A participação do enfermeiro se sobressai no desenvolvimento de habilidades técnicas e, sobretudo, na orientação aos usuários dos serviços de saúde e à equipe de enfermagem acerca das ações desempenhadas, de modo a ampliar o conhecimento, os argumentos científicos, além da humanização da atenção prestada, a fim de promover a qualidade da assistência.

Acredita-se que esta pesquisa poderá contribuir para direcionar ações de cuidado individualizadas para responder às necessidades das mães e seus $\mathrm{RN}$, uma vez que os diagnósticos encontrados permitem o delineamento das ações de cuidado e o envolvimento não apenas da mãe e filho, mas também da família. 


\section{REFERÊNCIAS}

1. Ministério da Saúde. Política de aleitamento materno. Brasília, DF: O Ministério; 2008.

2. Tamez RN, Silva MJP. Enfermagem na UTI neonatal: assistência ao recém-nascido de alto risco. 4. ed. Rio de Janeiro: Guanabara-Koogan; 2009.

3. Matos TA, Souza MS, Santos EKA; Velho MB, Seibert ERC, Martins NM. Contato precoce pele a pele entre mãe e filho: significado para mães e contribuições para a enfermagem. Rev Bras Enferm 2010; 63(6): 998-1004.

4. Amorim MM, Andrade ER. Atuação do Enfermeiro no PSF sobre Aleitamento Materno. Perspectivas [ periódico na internet]. 2009 [acesso em 21 out 2009];3(9). Disponível em: http://www.perspectivasonline.com.br

5. Almeida NAM, Fernandes AG, Araújo CG. Aleitamento materno: uma abordagem sobre o papel do enfermeiro no pós-parto. Rev Eletr Enf [periódico na internet]. 2004 [acesso em 20 out 2009];6(3):358-67. Disponível em: http://www.fen.ufg.br

6. Becker TAC, Teixeira CRS, Zanetti ML. Diagnósticos de enfermagem em pacientes diabéticos em uso de insulina. Rev Bras Enferm 2008; 61(6): 847-52.

7. Nóbrega MML, Garcia TR. Perspectivas de incorporação da Classificação Internacional para a Prática de Enfermagem (CIPE) no Brasil. Rev Bras Enferm 2005;58(2):27-30.

8. Braga CG, Cruz DALM. A Taxonomia II proposta pela North American Nursing Diagnosis Association (NANDA). Rev Latino-Am Enferm 2003;11(2):40-4.

9. Abrão ACFV, Gutierrez MGR, Marin HF. Diagnóstico de Enfermagem amamentação ineficaz - Estudo de identificação e validação clínica. Acta Paul Enferm 2005; 18(2):46-55.

10. NANDA Internacional. Diagnósticos de Enfermagem da Nanda: definições e classificação 2009-2011. Porto Alegre: Artmed; 2010.

11. Foschieira F, Vieira CS. O diagnóstico de enfermagem no contexto das ações de enfermagem: percepção dos enfermeiros docentes e assistenciais. Rev Eletr Enf [periódico na Internet]. 2004 [acesso em 18 out 2009];6(2):189-98. Disponível em: http://www.fen.ufg.br

12. Alves AR, Chaves EMC, Freitas MC, Monteiro ARM. Aplicação do processo de enfermagem: estudo de caso com uma puérpera. Rev Bras Enferm 2007;60(3):344-7.

13. Inácio CCN, Chaves EMC, Freitas MC, Silva AVS, Alves $A R$, Monteiro AR. Diagnósticos de enfermagem em unidades de alojamento conjunto. Rev Bras Enferm 2010;63(6):894-9.

14. Horta WA. O processo de enfermagem. São Paulo: EPU; 1979

15. Azevedo DS, Reis ACS, Freitas LV, Costa PB, Pinheiro PNC, Damasceno AKC. Conhecimento de primíparas sobre os benefícios do aleitamento materno. Rev Rene 2010;11(2):53-62.

16. Marques MCS, Melo AM. Amamentação no alojamento conjunto. Rev CEFAC 2008;10(2):261-71.

17. Figueiredo MG, Sartorelli DS, Zan TAB, Garcia E, Silva LC, Carvalho FLP, Pascotto RC,et al. Inquérito de avaliação rápida das práticas de alimentação infantil em São José do Rio Preto, São Paulo, Brasil. Cad Saúde Pública 2004;20(1):72-9.

18. Davim RMB, Enders BC, Silva RAR. Sentimentos de mães ao amamentarem seus bebês prematuros em sistema de alojamento conjunto. Rev Esc Enferm USP 2010;44(3):713-8.

19. Rivemales MC, Azevedo ACC, Bastos PL. Revisão sistemática da produção científica da enfermagem sobre o desmame precoce. Rev Enferm UERJ 2010;18(1):132-7.

20. Ferreira HS, Vieira EDF, Cabral Junior CR, Queiroz MDR. Aleitamento materno por trinta ou mais dias é fator de proteção contra sobrepeso em pré-escolares da região semiárida de Alagoas. Rev Assoc Med Bras 2010;56(1):74-80.

21. Vieira F, Bachion MM, Salge AKM, Munari DB, Souza MM. Diagnósticos de enfermagem da Nanda no período pós-parto imediato e tardio. Esc Anna Nery Res Enferm 2010;14(6):83-9. 